\title{
ON THE ACCELERATION PROCESSES IN SOLAR FLARES
}

\author{
Z. ŠVESTKA \\ American Science and Engineering, Cambridge, Mass. 02139, U.S.A.*
}

\begin{abstract}
The paper summarizes what we know about the acceleration processes on the Sun. Four different instabilities are distinguished: (1) One with purely thermal consequences giving rise to the origin of any flare. (2) A non-thermal process at the flash phase of flares giving rise to $\sim 100 \mathrm{keV}$ electrons and protons, manifested through hard X-ray and impulsive microwave bursts (current interruption?). (3) An instability giving rise to streams of electrons, without accelerating protons, manifested by type III bursts (tearing-mode instability?). When (2) and (3) are linked, flare associated electron events in space are often recorded. (4) Finally an explosive instability produces a shock wave which manifests itself as a type II burst. This instability leads to a second-step acceleration of particles preaccelerated in (2) and gives origin to $>10 \mathrm{MeV}$ protons and relativistic electrons (probably stochastic acceleration).
\end{abstract}

I intend to summarize what we know about the acceleration processes in solar flares. When doing that we must distinguish very carefully what we know for sure, what we believe to know with some degree of certainty, and what we simply guess. Unfortunately there are only very few pieces of knowledge that we know for sure.

First of all we know that the majority of flares only emit kinds of radiation which can be completely interpreted as being thermal. Figure 1 shows an example of such a typical flare. There is no hard X-ray component present which would need an explanation through non-thermal processes. All the radiation we receive from such a flare can be interpreted as due to increased temperature in the solar corona to some 20 or $30 \times 10^{6} \mathrm{deg}$, admitting that there may be cores of higher temperature which we average when making a temperature estimate.

This leads to the first important conclusion, namely that the basic instability which gives rise to a flare must be a thermal instability**. If we make the reasonable assumption that energy losses are mainly due to losses through radiation, we can get some information on the height of the origin of this instability. As it is well known, the radiative losses increase with increasing temperature up to some $500000 \mathrm{~K}$ but they become smaller if temperature further increases. This means that the instability which gives rise to a flare should take place higher than the layer with $500000 \mathrm{~K}$ temperature, i.e., the flare must originate in the transition layer or higher in the corona. Only there, if some additional energy is put in and temperature increases, this leads to decreasing losses of energy and consequently further increase in temperature.

These conclusions are pretty sure but not yet definite. There is one observation which seems to contradict the thermal nature of the X-ray flare radiation and that is the observation of the polarization of X-rays. One would expect that X-rays are polarized if they are due to directed beams of electrons which happens during a non-thermal

* This lecture was prepared while working on a grant of Stifterverband für die Deutsche Wissenschaft at Fraunhofer Institute, Freiburg.

** By this term we mean any instability, the consequences of which are purely thermal. 


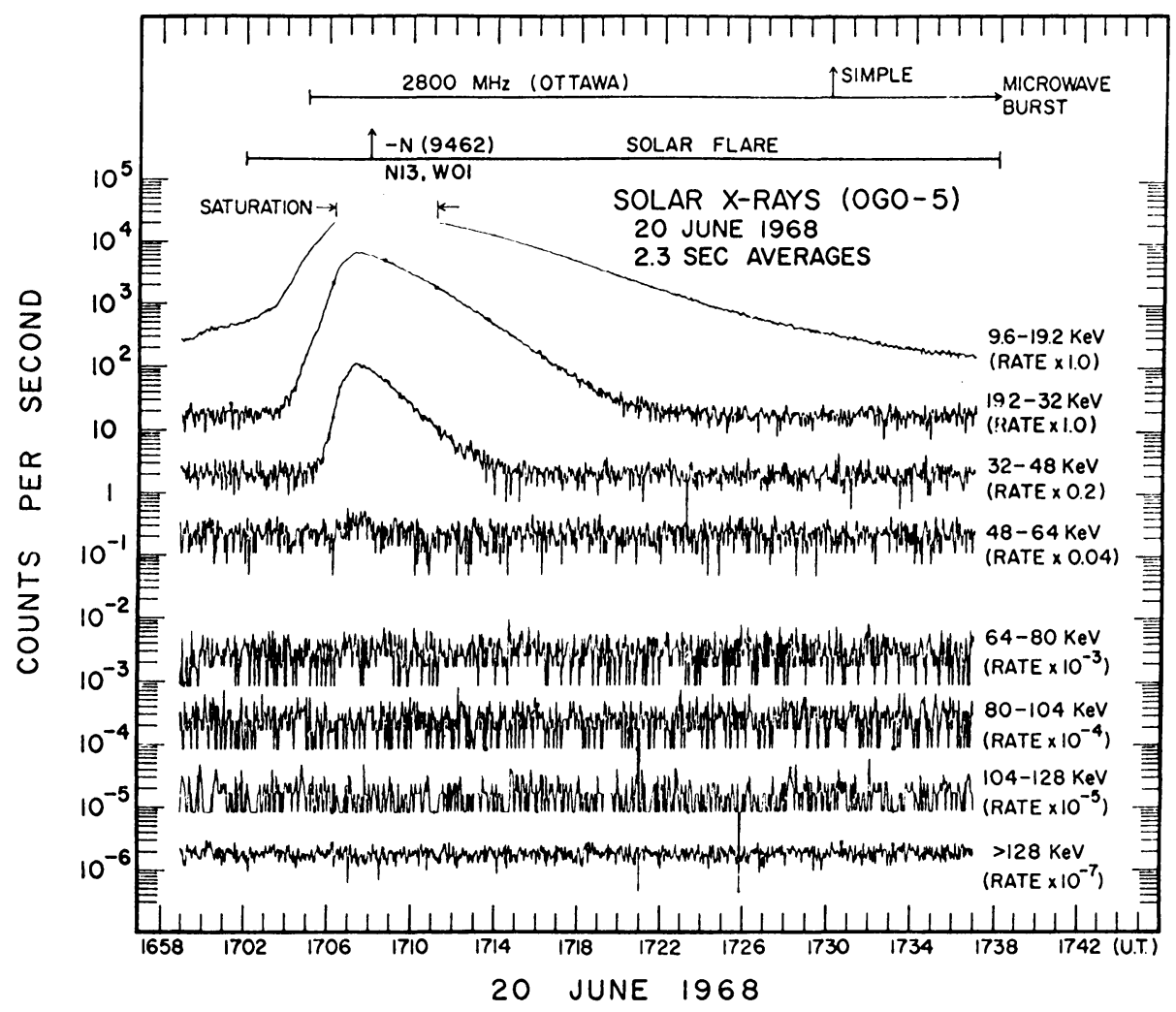

Fig. 1. A thermal X-ray flare burst, without any impulsive component. (After Kane, 1969; the increase at relatively high energies is due to pile-up and probably corresponds to $\mathrm{X}$-rays below $10 \mathrm{keV}$.)

acceleration process. Such a process as we shall see is fairly short-lived and therefore also the polarization should last for only a very short time. The observations, however, show that polarization of X-rays in flares lasts for many, even tens of minutes (Tindo et al., 1972; Thomas, 1975). This may be interpreted as due to continuous non-thermal acceleration of particles in the flare region so that the radiation only seems to be thermal but actually it is produced by a continuous nonthermal acceleration process. I mention this only to show that even this conclusion is open to doubt. Actually I believe that we are dealing here with thermal radiation. The observations of polarization are still fairly uncertain and even if one accepts these results of polarization measurements as real, there is still a possibility, e.g., to explain them as due to heat conduction in strong magnetic fields. In strong fields the heat conduction follows strictly the magnetic field lines and therefore we may encounter even in this case something like directed streams of particles.

Let us assume therefore that our first conclusion is correct. The flare originates through a thermal instability and in most flares we do not need any other process; only increase and subsequent decrease of temperature in the solar corona which affects 


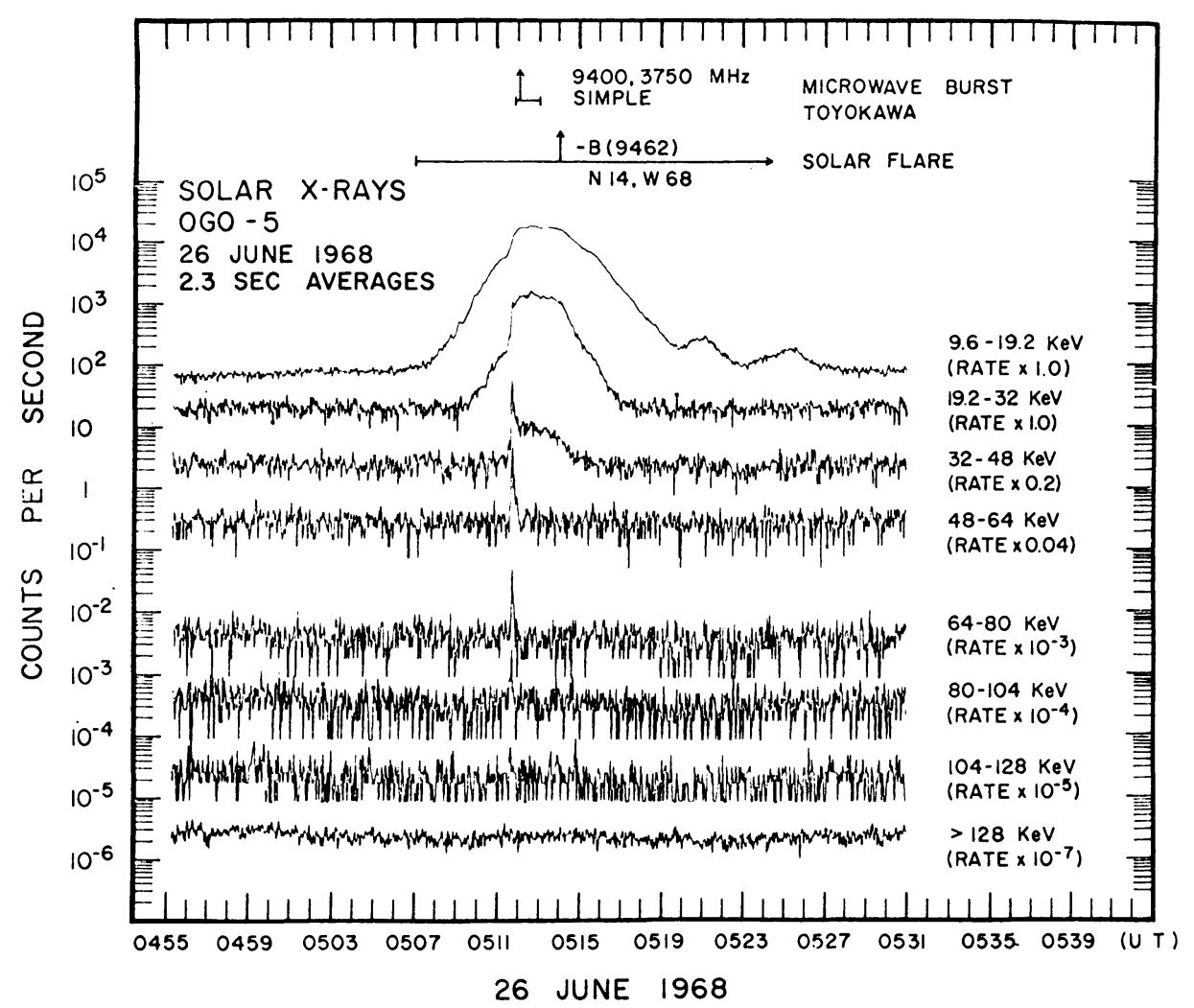

Fig. 2. An impulsive non-thermal hard X-ray burst occurring after the onset of the thermal flare. (After Kane, 1969.)

lower layers of the atmosphere through heat conduction. However, in many flares we observe something more: We observe, as Figure 2 shows, a short-lived emission in hard $\mathrm{X}$-rays during the onset phase of the flare development. This hard X-ray phase can be very short in some flares, just tens of seconds, but in larger flares it may last for a few minutes. All this occurs before the flare maximum in the $\mathrm{H} \alpha$ line, that means during the flash phase of the flare, and it mostly starts after the onset of the soft X-ray emission, that means after the onset of the basic thermal flare phenomen. In some cases the hard X-ray origin and the soft X-ray origin coincide but there is no case when the hard-X-rays would start before the soft X-ray emission; therefore, we can conclude that the thermal instability we mentioned before is the basic instability which gives origin to all kinds of flares.

However, in the flares of the type demonstrated in Figure 2, we meet with an additional process which obviously is of non-thermal nature. It manifests itself through hard X-rays and impulsive microwave bursts. Both of these phenomena can be interpreted as due to streams of electrons with power-law spectrum in energy (Takakura and Kai, 1966: Kane and Anderson, 1970). These electrons produce hard X-rays 
through bremsstrahlung and impulsive microwave bursts through gyrosynchrotron radiation. Again, it might be possible to interpret these bursts as thermal ones (Kahler, 1975). However, the type of the spectrum, the impulsive behavior and the fact that in some cases very high energy particles coming from these sources are reported in space, are very much in favor of the non-thermal interpretation.

Thus we meet already with two different instabilities in flares. The first basic thermal instability giving rise to all kinds of flares, and the non-thermal instability which occurs in some flares shortly after the onset during their flash phase. The hard X-ray and impulsive microwave bursts give evidence only on acceleration of electrons to energies up to a few hundred of $\mathrm{keV}$. We do not know whether protons are also accelerated at the same time because protons of these energies do not produce any effect that might be observed. We can only suppose that protons are accelerated by the same process as well and the argument for it is that we need the protons to be preaccelerated and thus prepared for another acceleration process to much higher energies which is observed in some rare cases as we shall see later on.

In any case this acceleration process is a weak one accelerating particles only to energies below or close to $1 \mathrm{MeV}$. It is of interest to see that the flares which contain this non-thermal component, that is in which this non-thermal acceleration process takes place, occur in special locations on the Sun. Most often we see them in active regions which are magnetically complex. That means in active regions where a new magnetic flux emerged and penetrated into an older existing one, thus producing a very complex magnetic structure in the region. Flares which occur in such magnetically complex active regions are much more likely to be associated with a non-thermal acceleration process than flares which occur in bipolar magnetic configurations. Another interesting feature is that flares which occur at about the same location produce very similar hard X-ray and impulsive microwave bursts; for example, radio bursts with maximum flux at about the same frequency (Švestka et al., 1974). This shows that the non-thermal instability is about the same in them and it can repeat several times during one or two days in flares which appear at about the same place in the active region.

Another manifestation of a non-thermal acceleration process on the Sun is the very well-known type III burst (cf. Lin, 1975). It is a fast drifting radio burst produced by a stream of electrons which propagate with a speed which is a significant fraction of the velocity of light. Many years ago De Jager and Kundu (1963) suggested that type III bursts and the microwave impulsive bursts or hard X-ray bursts are produced by the same instability which just accelerates electrons to high velocity. Those electrons which propagate downwards produce impulsive microwave bursts and hard X-ray bursts and those electrons which propagate upwards produce type III bursts. However, it appears that the situation is not that simple. First of all, when we try to correlate type III bursts and microwave bursts, we find that an exact time coincidence can be proved in less than $25 \%$ of cases (Švestka and Fritzová, 1974; Kane, 1972). In some other cases the coincidence may still exist since there is a series of type III bursts which lasts during the period when the microwave burst occurs. But there are 
at least $50 \%$ of cases when the type III burst occurs clearly at another time than the microwave burst or when only one of these kinds of bursts is observed. Apart from it the microwave bursts are closely and always associated with flares in contradistinction to the type III bursts of which only some $30 \%$ are associated with flares or sub-flares (Švestka, 1975).

The majority of type III bursts appear without any flare; on the other hand they are obviously restricted to particular active regions and to a particular phase of development of these active regions. There may be a large well-developed active region on the Sun which does not produce any type III bursts at all. Another region also inactive in type III bursts suddenly starts to produce type III bursts in large quantity, maybe several tens per day, and this production again suddenly stops after $20 \mathrm{~h}$ or one or two days. So far we are unable to find what makes particular active regions productive in type III bursts.

Figure 3a shows the dependence of the daily number of occurrences of microwave bursts on the daily flux value of radio emission of the Sun at $2800 \mathrm{MHz}$. This emission characterizes the importance of the solar activity and in particular it increases when magnetically complex active regions are present on the solar disk. We see that the occurrence of microwave bursts increases with the increasing radio flux as it is to be expected because microwave bursts predominantly occur in magnetically complex active regions. On the other hand, Figure $3 \mathrm{~b}$ shows that no such dependence does exist for type III bursts and indeed type III bursts do not prefer magnetically complex active regions.

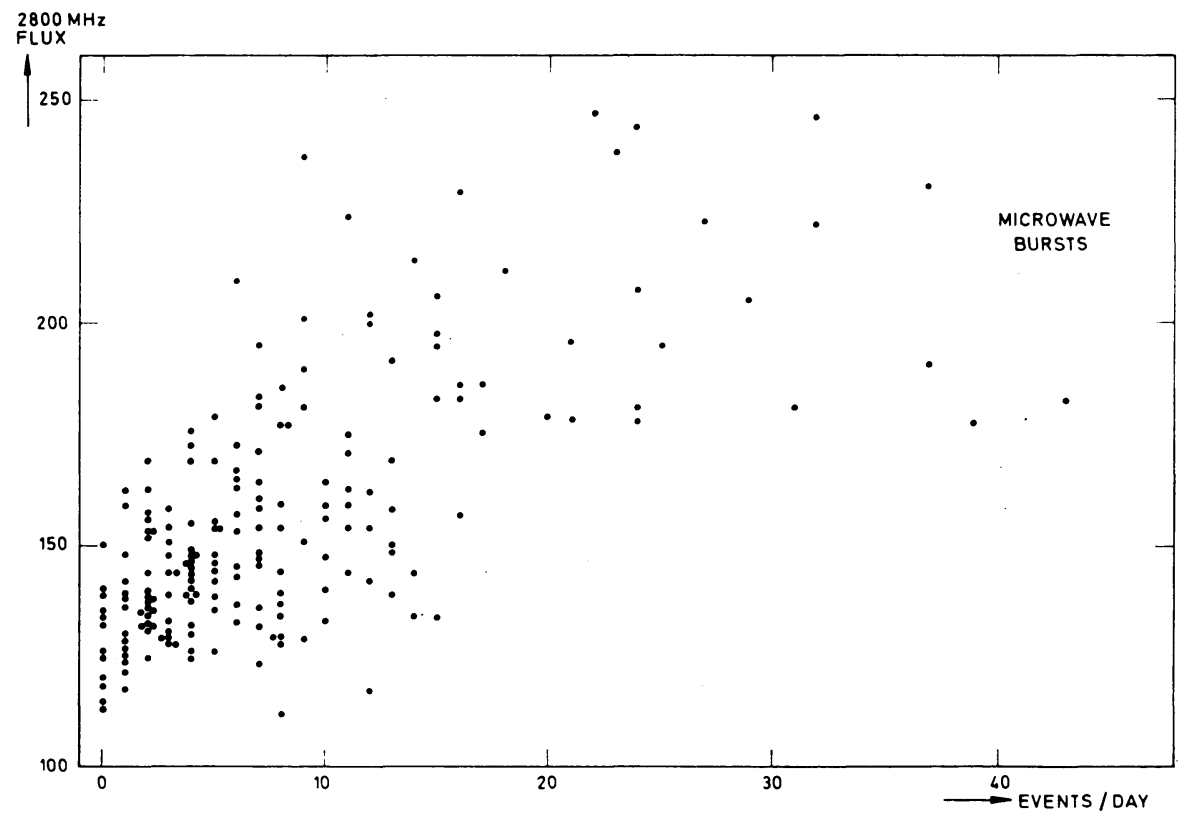

Fig. 3a. Dependence of the daily number of occurrences of microwave bursts on the daily flux value of radio emission of the Sun at $2800 \mathrm{MHz}$. 


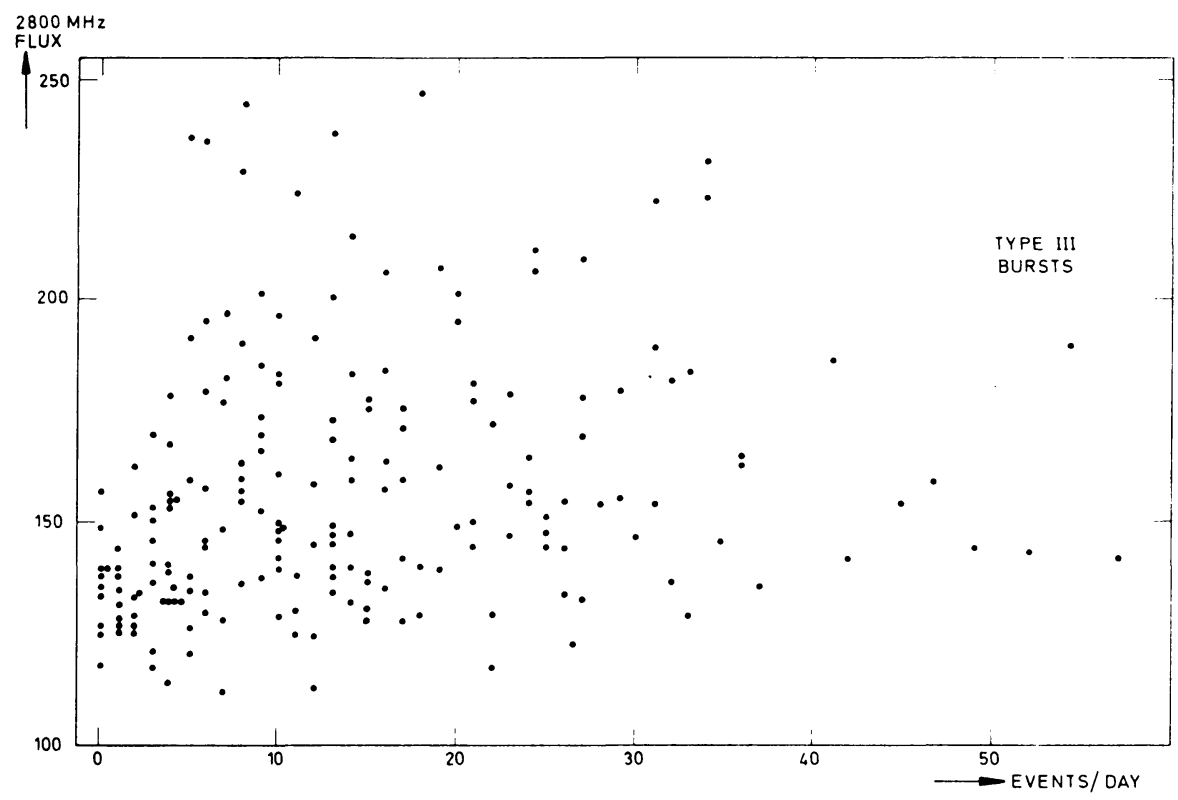

Fig. 3b. The same dependence as in Figure 3a, plotted for the daily number of occurrences of type III bursts.

All these differences in the type III and microwave burst occurrence indicate that we meet actually with two different acceleration processes on the Sun. One of them, always associated with flares, gives rise to microwave and hard X-ray bursts; another one, only sometimes associated with flares, gives rise to the type III bursts. Only in relatively rare cases these two acceleration processes are linked. In that case we have simultaneously a hard X-ray burst, impulsive microwave burst and type III burst and such events are always flare associated. The type III bursts which coincide with hard $\mathrm{X}$-ray bursts and microwave bursts and occur in the onset phase of a flare are usually very strong and they are accompanied with the type $\mathrm{V}$ radio continuum in most cases. The electron stream which produces such strong type III bursts can be followed quite often on radio waves very far from the Sun, deep into space up to the distance of the Earth. Satellites around the Earth then discover these electrons and electron streams in space, the so-called pure electron events.

We know that the electrons present in these streams are of energies of a few tens of $\mathrm{keV}$ and that there are no protons with energies in excess of $300 \mathrm{keV}$. We do not know whether protons with energies of a few tens of $\mathrm{keV}$ are present in the stream because until very recently these particles have never been measured in space. Apart from it a correlation of the electron and proton occurrence is quite difficult. Electrons with energies of about $40 \mathrm{keV}$ need just $20 \mathrm{~min}$ to get from the Sun to the Earth but protons of the same energy need about $14 \mathrm{~h}$ to make this trip. Nevertheless, the fact that the $300 \mathrm{keV}$ protons are essentially always missing in these streams is a strong argument pointing to the conclusion that protons are not accelerated in these cases. That means 
that the acceleration process which gives rise to the type III burst and electrons in space doesn't accelerate protons to energies comparable to the energies of electrons.

Hence we meet already with three different instabilities in flares. First with a thermal instability (instability No. 1) which gives origin to all flares. Second, with a nonthermal instability (instability No. 2) which accelerates electrons (and most probably also protons) to energies below or of the order of $1 \mathrm{MeV}$ and which produces the hard $\mathrm{X}$-rays and impulsive microwave bursts. This instability occurs in some flares. Finally, there is an instability No. 3 which is not necessarily associated with flares but which is related to particular configurations in particular active regions. It produces streams of electrons most probably without protons (e.g. particles are not accelerated to the same energy but to the same rigidity) and these streams of electrons manifest themselves as type III bursts on the radio waves. Although these bursts usually occur in active regions without any accompanying flare phenomenon, they tend to be associated with flares if flares occur in these particular active regions. In that case quite often the two acceleration processes giving origin to microwave bursts and type III bursts are linked and coincide exactly in time.

Still none of these instabilities can give rise to particles of extremely high energies which we observe from time to time in space. Cases when relativistic electrons and protons in excess of $10 \mathrm{MeV}$ are observed in space are rather frequent and in infrequent but very important cases we observe protons up to energies of $1000 \mathrm{MeV}$. Naturally, they cannot be of thermal origin. The instability No. 2 gives rise to electrons of energies only up to $1 \mathrm{MeV}$ and even if we assume that protons are also accelerated by this process, they hardly would be accelerated to higher energies. Finally, the instability No. 3 does not produce any protons with energies in excess of $300 \mathrm{keV}$. Therefore, we obviously need still another instability (No. 4) in order to produce energetic particles of these extremely high energies. What do we know about this strong acceleration process?

When protons of high energies and relativistic electrons are recorded in space we observe in the majority of cases a type IV burst on the radio waves, that is a continuum emission within the whole band of frequencies produced through gyrosynchrotron radiation of mildly relativistic electrons. Thus the type IV burst gives an evidence that mildly relativistic electrons have been accelerated in the region.

In essentially all such events we also observe the instability No. 2 , that means we observe hard X-ray bursts and impulsive microwave bursts which usually form the first phase of the subsequent type IV burst. Therefore, several years ago De Jager (1969) suggested that relativistic electrons and high energy protons are accelerated in a second acceleration step following our instability No. 2. This supposition was a natural consequence of theoretical difficulties which one had with acceleration of protons to very high energies starting with the energies which protons have in a thermal flare. If the protons are first preaccelerated in the first acceleration step, it is much easier then to accelerate them further in a second acceleration step to higher energies.

This conception of two steps of acceleration is most probably correct. Since the metric type IV burst is sometimes delayed for minutes or tens or minutes, De Jager 
supposed that this second acceleration step would follow some ten minutes or maybe even later after the first acceleration process which gives rise to particles with energies of about $1 \mathrm{MeV}$. However, there are two observations which indicate very strongly that the second acceleration step must follow immediately the first one. One is the observation of white light flare emission which as we believe is produced by high energy particles impinging on the photosphere and which occurs essentially at the same time as the hard X-ray and microwave bursts (Švestka, 1970; McIntosh and Donnelly, 1972; Rust and Hegwer, 1975). We need protons with energies in excess of some $20 \mathrm{MeV}$ to produce this emission so they must be produced in the second acceleration step. A similar result follows from gamma-ray observations. In the 1972, August 4 flare Chupp et al. (1973) and in the 1972, August 7 flare Vedrenne (1975) recorded the gamma-ray line produced by neutrons on the Sun and these neutrons need nuclei with energies in excess of $30 \mathrm{MeV}$ to be produced. Therefore, the occurrence of this line again needs a second step of acceleration to be accomplished and similarly to the white light emission the gamma-rays occurred essentially simultaneously* with the hard X-ray burst. This leads to a definite conclusion that if the high energy protons and relativistic electrons are accelerated in the second step of acceleration, this second step must follow within one minute or less the first acceleration process.

We have seen that both the rather weak acceleration processes discussed before manifested themselves quite clearly on the radio waves as microwave bursts or type III bursts respectively. Therefore, one can suppose that the much stronger second-step acceleration process should manifest itself in some pretty obvious way as well. One of its manifestations of course is the type IV burst, but this is only a consequence of the process. Many high-energy electrons accelerated in the second step are trapped in the solar corona and they become visible through gyrosynchrotron radiation as the type IV burst. This emission can be delayed so that the type IV burst does not show us the actual time of occurrence of the acceleration. There is however one more type of radio bursts which may be directly associated with the second step process and this is the type II burst.

The idea that type II bursts which are produced by shock waves propagating through the corona may be closely associated with the acceleration of high energy protons is not new. It was expressed several times ago and demonstrated for a few individual events (e.g., Frost and Dennis, 1971). But statistics so far failed to prove any clear association between type II bursts and strong particle events on the Sun. We have recently repeated the statistical discussion of this kind having the great advantage of the complete list of all particle events of different sizes published in the newly prepared Catalog of Solar Particle Events compiled by the Working Group 2 of the former Inter-Union Commission on Solar-Terrestrial Physics (Švestka and Simon, 1975). This very complete set of solar particle data, combined with greatly improved records of the radio emission by dynamic spectrographs (that means, improved as to the

* A delay of about $2 \mathrm{~min}$ is to be expected due to the line formation process on the Sun. 
sensitivity and as to the coverage in time) has made it possible to study the correlation between type II bursts and particle events on the Sun in full detail. This was not possible earlier when both particle data and type II burst observations were incomplete. This new statistical discussion (Švestka and Fritzová, 1974) has convinced us that indeed type II bursts are closely associated with the second acceleration step in flares.
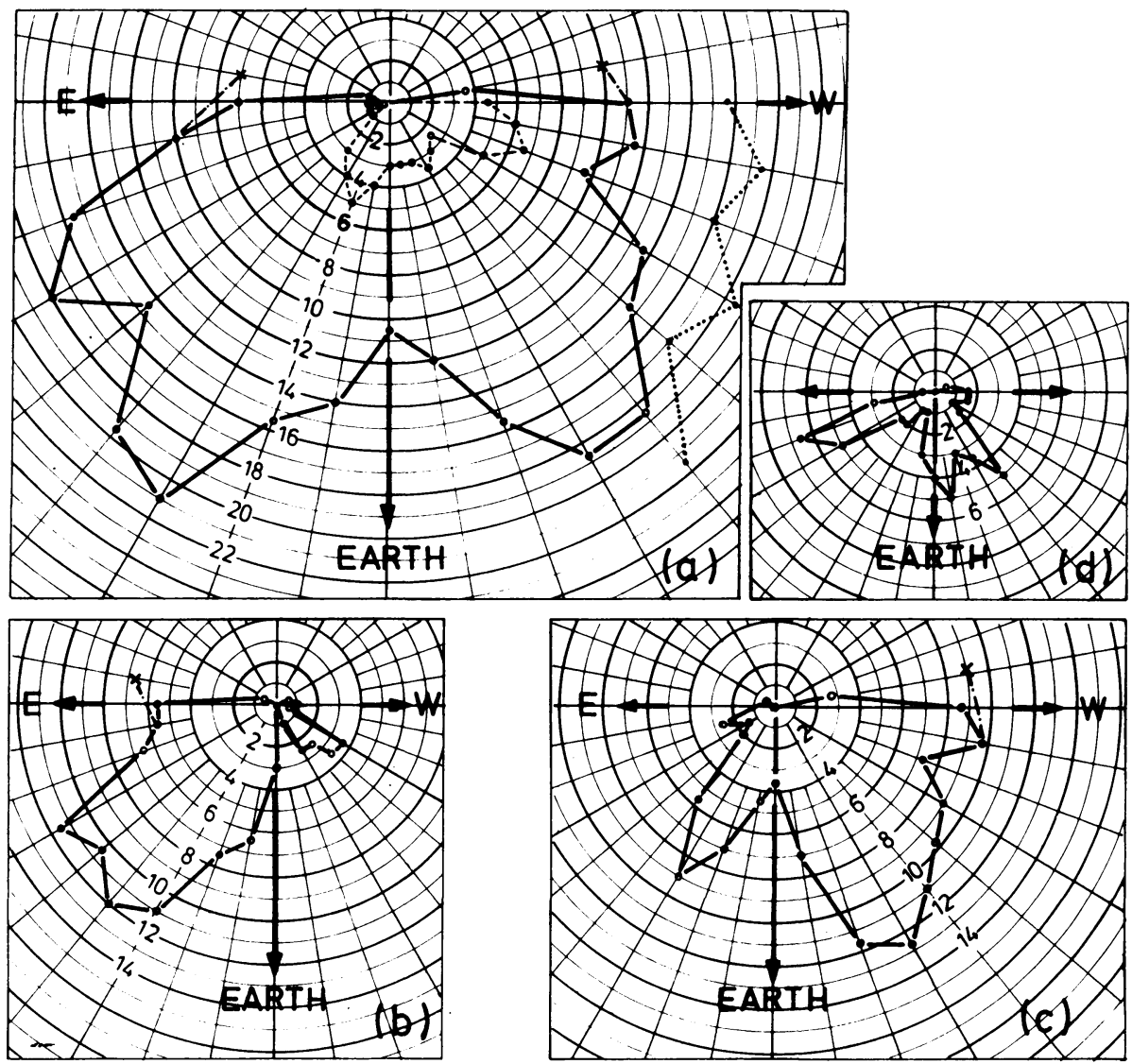

Fig. 4. The distribution in solar longitude of all type II bursts (heavy line in (a)), of those (c) that produced energetic particles and (b) that did not produce energetic particles near the Earth. (After Švestka and Fritzová-Švestková, 1974).

Figure 4a shows the distribution in solar longitude of all type II bursts during 30 months in the years 1966 to 1968. The position of each burst is identified with the position of the flare associated with it. Figure $4 \mathrm{c}$ shows the distribution in solar longitude of those type II bursts which were associated with particle events and Figure $4 \mathrm{~b}$ shows the distribution of those type II bursts which were not associated with any particle event. The graphs show quite clearly that the proton producing flares prevailed on the western hemisphere while at least $84 \%$ of type II bursts without particle events were on the eastern hemisphere. This is exactly what we expect if essentially all type II 
bursts are associated with acceleration of protons on the Sun and if particle propagation goes along the Archimedes spirals.

Figure 5 shows the distribution of time intervals between the maximum of the impulsive microwave burst and the type II burst onset, for metric type II bursts. The metric type II bursts start on an average $2.6 \mathrm{~min}$ after the maximum of the microwave burst. There are $20 \%$ of cases when the type II bursts originated prior to the microwave burst maximum, but there is no case at all when type II bursts would have started

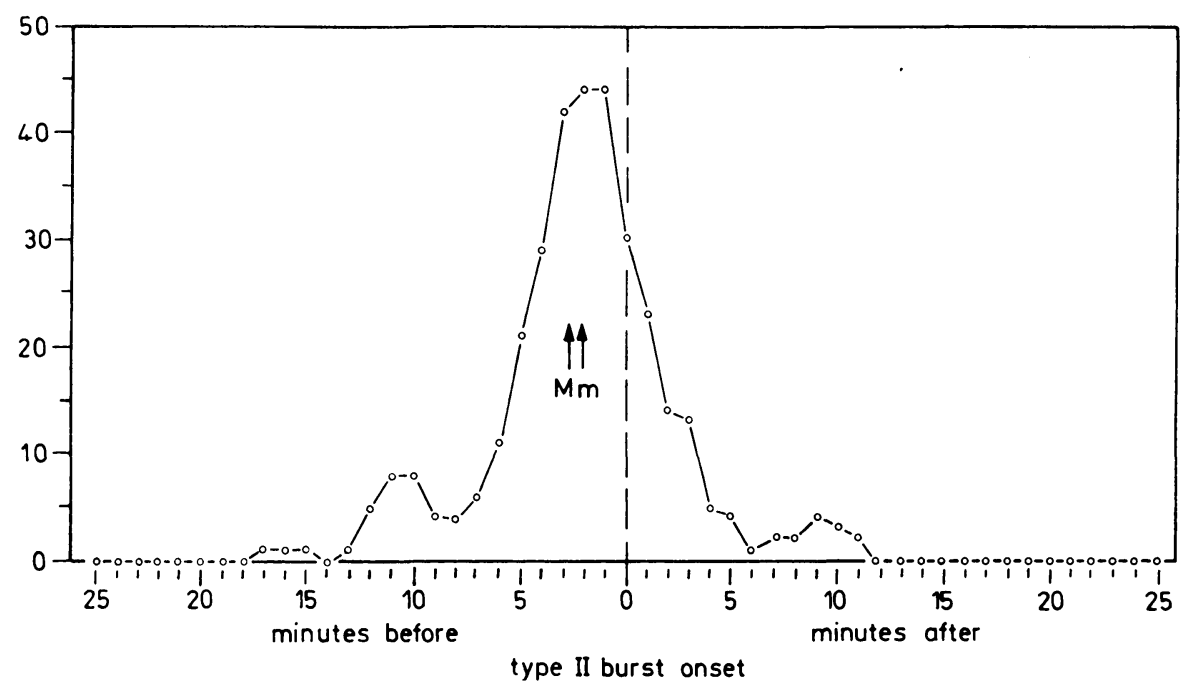

Fig. 5. The distribution of time intervals between the maximum of the impulsive microwave burst (i.e. maximum or end of the first step acceleration phase) and the metric type II burst onset. $M$ is the mean, and $m$ the median time interval.

before the onset of the microwave burst. Therefore, the type II burst obviously originates close to the time of the primary acceleration process which gives rise to the nonrelativistic electrons which produce the impulsive microwave and hard X-ray bursts. If we assume as the statistical average that the type II originates close to the time of the microwave burst maximum, the $2.6 \mathrm{~min}$ difference means that the shock wave producing it propagates with the speed of about $1500 \mathrm{~km} \mathrm{~s}^{-1}$ which is an acceptable value.

We have found that the association of type II bursts with particles increases with the importance of the particle event. For polar cap absorption events at least $73 \%$ and potentially as many as $100 \%$ of the events were preceded by a type II burst. For particle events in which the proton flux in excess of $10 \mathrm{MeV}$ exceeded 1 proton $\mathrm{cm}^{-2} \mathrm{~s}^{-1}$ $\mathrm{s}^{-1}$ as many as $91 \%$ of proton events still might have been associated with type II bursts. On the other hand the association is sure for only $15 \%$ of cases if the flux of protons with energies above $10 \mathrm{MeV}$ is lower than 0.01 protons $\mathrm{cm}^{-2} \mathrm{~s}^{-1} \mathrm{sr}^{-1}$, and no association has been found for the pure electron events. This preference of strong proton events indicates that the type II bursts indeed characterize the second acceleration step in which the energy of the primarily accelerated particles is further increased 
It appears to be a reasonable assumption that this strong second step acceleration process is due to stochastic acceleration in turbulent plasma. Either the shock wave itself as it crosses the magnetic field lines or another effect produced by the same explosive instability gives rise to a powerful plasma turbulence in which stochastic acceleration of the particles preaccelerated in the first acceleration step can be accomplished. The short time differences between the maximum of the impulsive microwave burst and the onset of the metric type II burst, and in particular the fact that in some $20 \%$ of the cases the type II is observed even prior to the microwave burst maximum, indicates that the two steps of acceleration can be accomplished essentially simultaneously. While some particles are still being preaccelerated, other particles preaccelerated earlier already enter into the second acceleration step.

The first acceleration step is clearly characterized by the hard X-ray burst and impulsive microwave burst. These effects are essentially always observed (with the exception of limb events) when high energy particles are reported in space. On the other hand we know several strong and important particle events, like the cosmic-ray flare of 1966, July 7, or the flare of 1966, September 2, with 13 dB PCA effect, when no type III burst was observed at all. This fact, as well as the very frequent occurrence of type III bursts without any flare and the strong indication that protons are not accelerated in this kind of instability, gives an evidence that the type III burst type of acceleration is not identical with the primary acceleration step.

This leads us back to the problem whether protons are also accelerated in our acceleration processes 2 and 3 . We have seen that there is a rather strong evidence that protons are not accelerated through the instability giving rise to the type III bursts. On the other hand nothing contradicts the supposition that protons are accelerated through the process which gives origin to the electrons which produce the hard X-rays and impulsive microwave bursts. We have no direct evidence for it. However, as soon as we suppose that this acceleration process, our No. 2, is the first step in the acceleration which gives rise to high energy protons, we must necessarily assume that protons are preaccelerated through this process as well.

While we can make a reasonable assumption that stochastic acceleration is associated with the shock wave, it is difficult to say with our present knowledge which type of instability accelerates the particles in the first acceleration step and in the type III bursts. We can only guess here. It seems that the most acceptable mechanisms for these two acceleration processes are current interruption in the case of the first acceleration step and tearing mode instability in the case of the type III bursts. There are no strong arguments for it. But, current interruption seems to be an attractive mode of particle acceleration and we know that it is difficult, maybe impossible, to accelerate particles through this mechanism to high energies. However, it still seems to be within reasonable possibilities to accelerate particles through current interruption to energies of the order of $100 \mathrm{keV}$ as we observe. The tearing mode instability originally proposed by Sturrock (1968) for explanation of the whole flare phenomenon seems to be very appropriate for a type III burst because its duration is very short and 
in Sturrock's model it occurs high above the active region where type III bursts actually are observed.

Thus, summarizing, we have the following picture of the flare phenomenon: Somewhere in the transition layer or in the low corona an input of energy occurs which gives rise to a thermal instability. This thermal instability produces what we call a flare. In the majority of cases nothing more happens. Temperature increases, reaches a maximum and then the flare volume cools. Through heat conduction we observe the typical chromospheric flare. In some cases in addition to this thermal instability a current interruption occurs which accelerates electrons and protons to energies of a few hundred $\mathrm{keV}$. In these cases we observe hard $\mathrm{X}$-rays and impulsive microwave bursts. Apart from this kind of flare-associated instability there also exists another kind of instability, a tearing mode instability in coronal current sheets which gives rise, under conditions which we are unable to specify at the present time, to streams of electrons propagating up into the corona and giving rise to the type III bursts. In some particular cases these two instabilities are linked so that current interruption lower in the atmosphere and tearing mode instability in the corona occur at the same time. Then we have streams of electrons descending in the solar atmosphere and producing hard $\mathrm{X}$-ray bursts and impulsive microwave bursts and another stream of electrons going up into the corona and producing type III bursts. This electron stream can propagate very deep into space and produce pure electron events observed aboard satellites near the Earth. Finally, in some relatively few flares still another kind of instability accompanies the current interruption and this instability gives rise to a shock wave and strong turbulent motions in the atmosphere. In that case the particles, both electrons and protons, preaccelerated through the current interruption, may be accelerated to substantially higher energies up to tens or hundreds of $\mathrm{MeV}$ and we observe then the so-called proton events in space.

One could try, of course, to simplify this picture. The instabilities No. 2 and $3 \mathrm{might}$ be considered generally for one type of instability which behaves differently in different magnetic configurations, manifesting itself once through a microwave burst, once through a type III burst, and on rare occasion through a simultaneous occurrence of both these phenomena. However, the fact that we need protons to be accelerated at the time of the microwave bursts, while no protons are recorded in the type III streams, makes this simplification unlikely.

Similarly, one might suppose that the instability No. 4 which gives rise to the shock wave, is simply a strong fully developed instability No. 2 . One cannot exclude this possibility; however, the energy involved in the shock is pretty large, and thus it seems that something qualitatively different happens in the flares that produce the shock waves.

I am fully aware of the highly speculative nature of some parts of my talk. The interpretations are difficult with our limited amount of knowledge. However, I have considered it useful to try to summarize the observations which give us some information on the acceleration processes in flares and to emphasize that we possibly need several different instabilities and several acceleration mechanisms in order to explain all the phenomena observed. 


\section{References}

Chupp, E. L., Forrest, D. J., Higbie, P. R., Suri, A. N., Tsai, C., and Dunphy, P. P.: 1973, Nature 241, 333.

De Jager, C.: 1969, in C. de Jager and Z. Švestka (eds.), Solar Flares and Space Research, p. 1.

De Jager, C. and Kundu, M. R.: 1963, Space Res. 3, 836.

Frost, K. J. and Dennis, B. R.: 1971, Astrophys. J. 165, 655.

Kahler, S.: 1975, This volume, p. 211.

Kane, S. R.: 1969, Astrophys. J. Letters 157, L139.

Kane, S. R.: 1972, Solar Phys. 27, 174.

Kane, S. R. and Anderson, K. A.: 1970, Astrophys. J. 162, 1003.

Lin, R. P.: 1975; This volume, p. 385.

McIntosh, P. S. and Donnelly, R. F.: 1972, Solar Phys. 23, 444.

Rust, D. M. and Hegwer, R.: 1975, Solar Physics 40, 141.

Sturrock, P. A.: 1968, in K. O. Kiepenheuer (ed.), 'Structure and Development of Solar Active Regions', IAU Symp. 35, 471.

Švestka, Z.: 1970, Solar Phys. 13, 471.

Švestka,.Z.: 1974, Solar Flares, D. Reidel Publ. Co., Dordrecht, Holland (in press).

Švestka, Z. and Fritzová-Švestková, L.: 1974, Solar Phys. 36, 417.

Švestka, Z. and Simon, P. (eds.): 1975, Catalog of Solar Particle Events, 1955-1969, D. Reidel Publ. Co., Dordrecht, Holland.

Švestka, Z., Castelli, J. P., Dizer, M., Dodson, H. W., McIntosh, P. S., and Urbarz, H.: 1974, Flares of 8 June, 1972, at $13^{\mathrm{h}} 19^{\mathrm{m}}$ and $13^{\mathrm{h}} 52^{\mathrm{m}}$ UT, CINOF Report, presented at the STP Symposium, Sao Paulo, June.

Takakura, T. and Kai, K.: 1966, Publ. Astron. Soc. Japan 18, 57.

Thomas, R.: 1975, This volume, p. 25.

Tindo, I. P., Ivanov, S. L., Mandelshtam, S. L., and Skurygin, A. I.: 1972, Solar Phys. 24, 429. Vedrenne, G.: 1975, This volume, p. 315. 